\title{
MODELLING AND EXPERIMENTAL INVESTIGATIONS OF DC ELECTRIC ARCS IN ARGON AND CARBON DIOXIDE
}

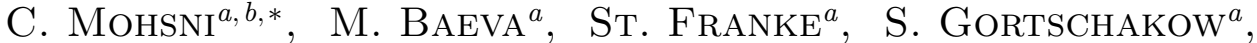 \\ D. Gonzalez ${ }^{a}$, Z. Araoud ${ }^{b}$, K. Charrada ${ }^{b}$ \\ ${ }^{a}$ Leibniz Institute for Plasma Science and Technology, Felix-Hausdorff-Str. 2, 17489 Greifswald, Germany \\ ${ }^{b}$ National School of Engineering of Monastir, 5000 Rue Ibn Jazzar, Monastir 5035, Tunisia \\ * mohsni_chayma@hotmail.fr
}

\begin{abstract}
In this work an arc model is employed along with electric and spectroscopic measurements to study DC electric arcs in $\mathrm{Ar}$ and $\mathrm{CO}_{2}$. The model is aimed at describing the arc and the electrodes. Simulation and experimental results are shown for currents between $150 \mathrm{~A}$ and $210 \mathrm{~A}$.
\end{abstract}

Keywords: thermal plasma, electric arcs, plasma-electrode interaction, plasma diagnostics.

\section{Introduction}

Experimental and simulation studies of electric arcs, which are used in industrial applications, are important for a deeper understanding of the arc process. Some gases such as $\mathrm{SF}_{6}$, used in circuit breakers, have a negative impact on the environment. Therefore, alternatives are considered and one potential candidate is carbon dioxide $\left(\mathrm{CO}_{2}\right)$ due to its electrical and chemical properties.

The modelling of electric arcs, which combines the equilibrium description of the bulk plasma and a nonequilibrium treatment of the near-electrode regions $[1,2]$, has been considered as an alternative to the fully equilibrium approach applied over the course of many years $[3,4]$ and the fully non-equilibrium approaches suggested more recently in $[5,6]$. The combined modelling of the plasma bulk and the cathode boundary layer in DC arcs with a refractory cathode allows accounting for the significant amount of electric power deposited in the thin boundary layer adjacent to the cathode, in particular for currents below $200 \mathrm{~A}$, and to reduce the discrepancy to the measured arc voltage, i.e. to improve the predictive capability of the model. This modelling approach can be, in general, more easily applied to molecular gases, which are used in industrial devices, e.g. circuit breakers.

In this work, $\mathrm{DC}$ arcs in argon and $\mathrm{CO}_{2}$ were studied. A Local Thermodynamic Equilibrium (LTE) arc model in $\mathrm{CO}_{2}$ was established while the model for argon is aimed at coupling the thermionic cathode, the LTE bulk plasma and the anode. To assess the model accuracy, electrical and spectroscopic measurements in various gases were carried out.

\section{Model description}

The development of the combined arc model includes two steps: the establishment of the LTE model of the arc column and the non-equilibrium model of the near-electrode regions. The LTE model is based on a magneto-hydrodynamic approach which involves the Navier-Stokes equations for conservation of mass, momentum and energy. The electrodes are included in the model and coupled to the plasma through a non-equilibrium boundary layer. It is assumed that the gas flow is laminar and the plasma behaves like a fluid which state is characterized by LTE. A stationary solution is sought for the equations

$$
\begin{gathered}
\nabla \cdot(\rho \mathbf{u})=0 \\
\rho(\mathbf{u} \cdot \nabla) \mathbf{u}=\nabla \cdot(-p \hat{I}+\hat{\tau})+\mathbf{F}_{\mathbf{L}} \\
\rho C_{p}(\mathbf{u} \cdot \nabla T)+\nabla \cdot \mathbf{q}=Q_{\mathbf{J}}-Q_{\mathrm{rad}}
\end{gathered}
$$

where $\rho$ is the mass density, $\mathbf{u}$ is the flow velocity, $p$ is the pressure, $\hat{\tau}$ is the stress tensor, $\hat{I}$ is the identity matrix, and $\mathbf{F}_{\mathbf{L}}=\mathbf{j} \times \mathbf{B}$ is the Lorentz force per unit volume. In Eq. (3), $C_{p}$ is the specific heat at constant pressure, $T$ is the gas temperature, $\mathbf{q}=$ $-\kappa \nabla T$ is the heat flux due to thermal conduction with $\kappa$ being the thermal conductivity. The gas is heated by Joule heating $Q_{\mathrm{J}}=\mathbf{j} \cdot \mathbf{E}$, where $j$ is the electric current density, and cooled by radiation $Q_{\mathrm{rad}}$. The Maxwell equations and the Ohm's law are used to obtain the self-induced magnetic field $\mathbf{B}$ and the electric potential $\varphi$

$$
\begin{aligned}
& \nabla \times \mathbf{B}=\mu_{0} \mathbf{j} ; \quad \mathbf{B}=\nabla \times \mathbf{A} \\
& \nabla \cdot \mathbf{j}=0 ; \quad \mathbf{j}=\sigma \mathbf{E}=-\sigma \nabla \varphi
\end{aligned}
$$

The electric field $\mathbf{E}$ and the magnetic field $\mathbf{B}$ couple the electromagnetic part of the model to the fluid through the Lorentz force per unit volume and the Joule heating. In Eq. (4) and (5), A is the vector potential and $\sigma$ is the electrical conductivity. The transport properties were provided by A. B. Murphy as calculated according to $[7,8]$ and applying a C-C collision integral from [9].

The heat conduction and current continuity equations are solved in the electrodes:

$$
\nabla \cdot\left(k_{\mathrm{s}} \nabla T\right)+\mathbf{j} \cdot \mathbf{E}=0
$$




$$
\nabla \cdot \mathbf{j}=0 ; \quad \mathbf{j}=\sigma_{\mathrm{s}} \mathbf{E}=-\sigma_{\mathrm{s}} \nabla \varphi
$$

The electrical conductivity $\sigma_{\mathrm{s}}$ and the thermal conductivity $k_{\mathrm{s}}$ of the solid materials are taken as a function of the temperature $T$. The cathode boundary layer includes the region of space-charge adjacent to the cathode surface (the sheath) and the ionization layer.

The energy balance in the boundary layer $[10,11]$ accounts for 1) the heat flux leaving the boundary layer and going to the cathode, 2) the electric power density deposited in the boundary layer, 3) the power brought by the electrons emitted from the cathode, and 4) the heat flux entering the boundary layer from the LTE plasma. The energy balance and the total current density, which is the sum of the density of the thermionic emission current $\left(j_{\mathrm{em}}\right)$, the ion current density $\left(j_{\text {ion }}\right)$, and the current density transported by counter-diffusing electrons $\left(j_{\text {be }}\right)$, determine the heat flux $q_{\mathrm{w}}$ and the current density $j_{\mathrm{w}}$ on the cathode surface as functions of the voltage drop $U_{\text {tot }}$ in the boundary layer and the surface temperature $T_{\mathrm{w}}$ :

$$
\begin{gathered}
q_{\mathrm{w}}\left(T_{\mathrm{w}}, U_{\text {tot }}\right)=j_{\mathrm{w}} U_{\text {tot }}-\frac{j_{\mathrm{w}}}{e}\left(A_{\mathrm{w}}-\Delta A_{\mathrm{w}}+3.2 k T_{\mathrm{e}}\right) \\
j_{\mathrm{w}}\left(T_{\mathrm{w}}, U_{\mathrm{tot}}\right)=j_{\mathrm{em}}+j_{\text {ion }}-j_{\mathrm{be}}
\end{gathered}
$$

Here, $A_{\mathrm{w}}$ is the work function of the electrode material, $\Delta A_{\mathrm{w}}$ is the Shottky correction, $T_{\mathrm{e}}$ is the electron temperature obtained from the boundary layer model. The voltage drop in the near-cathode layer includes the voltage drop in the space-charge sheath $\left(U_{\mathrm{d}}\right)$ and that in the ionization layer $\left(U_{\mathrm{i}}\right)$, i.e. $U_{\text {tot }}=U_{\mathrm{d}}+U_{\mathrm{i}}$. A similar approach has been applied in [12].

Losses due to black-body radiation of the hot body are considered as $q_{\mathrm{rad}}=\epsilon \sigma_{\mathrm{SB}} T_{\mathrm{w}}^{4}$. The resultant heat flux $q_{\mathrm{c}}=q_{\mathrm{w}}\left(T_{\mathrm{w}}, U_{\text {tot }}\right)-q_{\mathrm{rad}}$, and the current density $j_{\mathrm{w}}\left(T_{\mathrm{w}}, U_{\text {tot }}\right)$ serve as boundary conditions of equations (6) and (7). The cathode boundary layer is solved prior to the arc simulation for a series of values of the temperature on the cathode boundary $T_{\mathrm{w}}$ and the voltage drop $U_{\text {tot }}$. The results are provided as lookup tables and are used during the iterative solution of the combined model.

The consideration of the plasma-anode boundary layer is simpler [13]. The expression for the heat flux as in many LTE models $[3,4]$ is used. The normal component of the energy flux to the surface $q_{\mathrm{a}}$, which is added to the conductive one, is given by

$$
q_{\mathrm{a}}=-\epsilon \sigma_{\mathrm{SB}} T_{\mathrm{w}}^{4}+\left|j_{\mathrm{e}}\right| A_{\mathrm{an}}
$$

Here, $\epsilon$ is the emissivity of the surface, $\sigma_{\mathrm{SB}}$ is the Stefan-Boltzmann constant, $j_{\mathrm{e}}$ is the electron component of the current density and $A_{\text {an }}$ is the work function of the anode material. The term $\left|j_{\mathrm{e}}\right| A_{\mathrm{an}}$ accounts for heating of the anode due to condensation of electrons. For pure tungsten, the work function and the Richardson constant are $4.55 \mathrm{eV}$ and $0.602 \cdot 10^{6} \mathrm{Am}^{-2} \mathrm{~K}^{-2}$, respectively. The corresponding values for graphite are $4.6 \mathrm{eV}$ and $0.600 \cdot 10^{6} \mathrm{Am}^{-2} \mathrm{~K}^{-2}$. The model is set up on the commercial computational
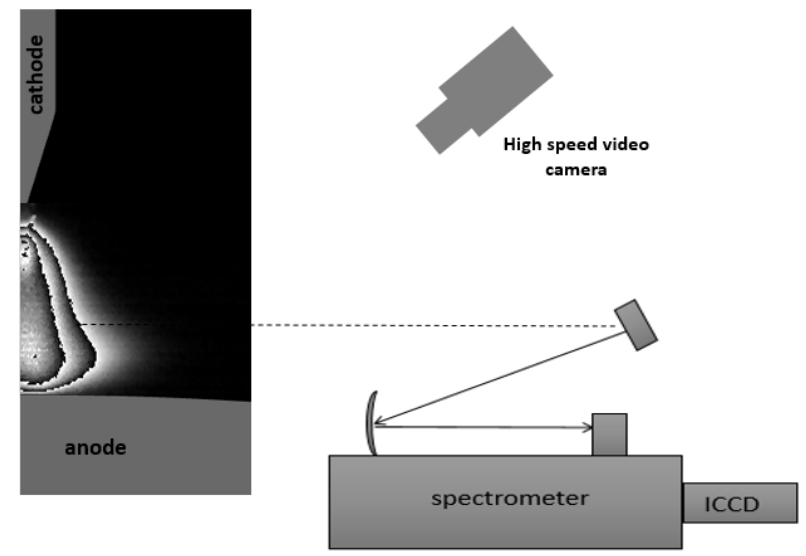

Figure 1. A view of the computational domain and the OES arrangement used for investigation of the arc.

platform COMSOL Multiphysics@ using the interfaces Laminar flow, Electric current, Magnetic field and Heat transfer. The computational domain includes both electrodes and the inter-electrode space in a radial extend of $30 \mathrm{~mm}$. A part of this domain is shown in Figure 1. Temperature of $300 \mathrm{~K}$, electric and magnetic insulation are set on the external boundaries. The anode is grounded. The total arc voltage drop in the boundary layer is adjusted until the integral of the current density along the cathode surface equals the desired arc current.

\section{Experimental setup}

A view of the spectroscopic equipment and the computational domain is shown in Figure 1. The setup is tested for arcs in argon and $\mathrm{CO}_{2}$. It comprises a spherically shaped anode, made of graphite and a cylindrical rod with a conical shape made of pure tungsten as cathode. The distance between the cathode tip and the anode is varied between 2 and $10 \mathrm{~mm}$.

The image of the arc is taken by a high-speed video camera of the type IDT Motion Pro Y6. The arrangement is placed inside a chamber which is filled with gas up to the desired pressure. The latter is controlled by a manometer. The arc is ignited making use of a spark coil arrangement. The equipment for optical emission spectroscopy (OES) consists of a spectrograph Acton SP2500 with a focal length of $0.5 \mathrm{~m}$ and an intensified CCD camera Princeton Instruments PIMAX4. Obtained images contain spectral and spatial side-on information about the arc. The arc is imaged by a spherical mirror on the entrance slit of the spectrometer with a width of $30 \mu \mathrm{m}$. Absolute calibration is performed by a tungsten strip lamp placed at the position of the arc.

The radiation of the Ar II line at $480 \mathrm{~nm}$ is spectrally resolved using a grating with 1800 lines $/ \mathrm{mm}$. The radiation of the O I line at $777 \mathrm{~nm}$ is resolved using a grating with 150 lines/mm. An integration over the wavelength range is performed and Abel inversion is done in order to get the radial dependence 


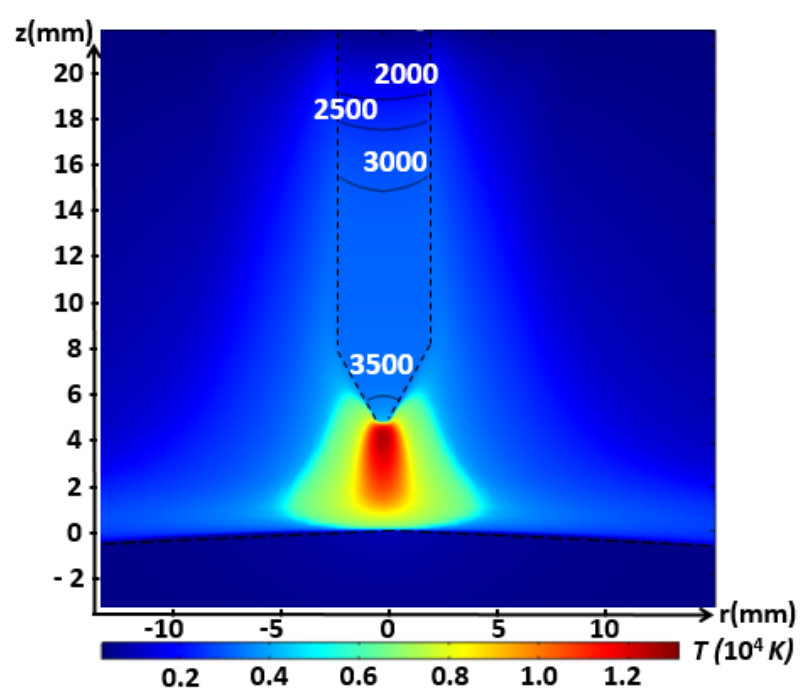

Figure 2. Two-dimensional distribution of the plasma temperature and the temperature in the electrodes obtained with the LTE model for an arc in $\mathrm{CO}_{2}$ at current of $200 \mathrm{~A}$.

of the emission coefficient. The number densities of the upper level corresponding to the transition of the Ar II $480 \mathrm{~nm}$, O I 777 lines are derived assuming LTE. The population density is related to the plasma temperature. The temperature is obtained from a pre-calculated equilibrium composition of argon and carbon dioxide.

\section{Results and discussion}

Calculations with the LTE arc model were performed for the arrangement shown in Figure 1 with $\mathrm{CO}_{2}$ at atmospheric pressure as working gas. Figure 2 shows the distribution of the temperature in the plasma and the electrodes for an arc current of $200 \mathrm{~A}$. Since a matching of the boundary layer with the LTE plasma is not considered, the temperature in the arc seems to

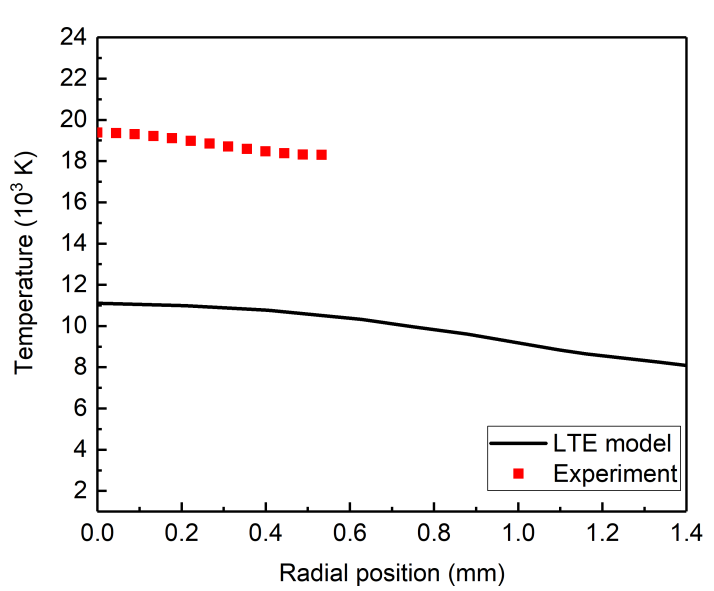

Figure 3. Radial plasma temperature obtained in the LTE model for an arc in $\mathrm{CO}_{2}$ and the plasma temperature from OES for an arc current of $200 \mathrm{~A}$ at axial position $1 \mathrm{~mm}$ away from the cathode tip.

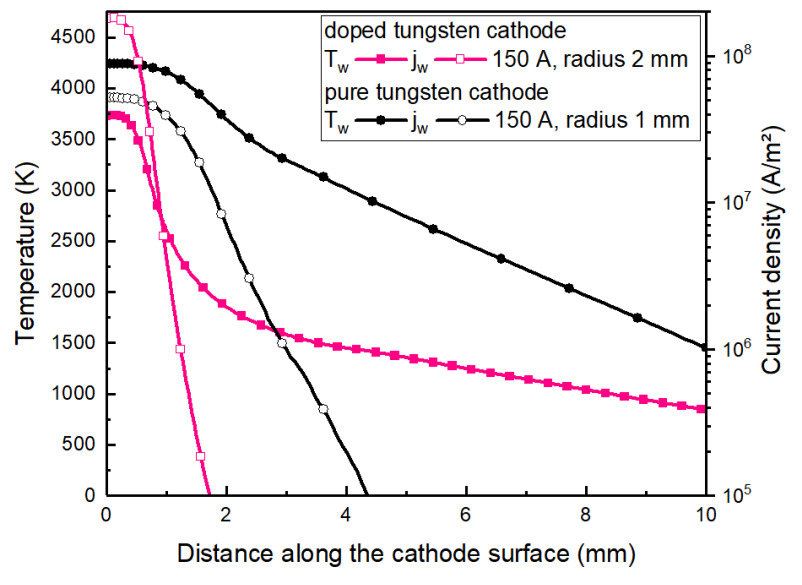

Figure 4. Temperature and normal current density along cathode surface for doped and pure tungsten cathodes for an arc current of $150 \mathrm{~A}$.

be underestimated as the experimental results show in Figure 3.

The combined model has been applied first for argon as working gas to obtain the plasma parameters for arc currents of $150 \mathrm{~A}$ and $210 \mathrm{~A}$. Figure 4 shows the temperature on the cathode surface and the current density for an arc current of $150 \mathrm{~A}$ for two cathode configurations: a) doped tungsten cathode (Ce-W) with a radius of $2 \mathrm{~mm}$, and $\mathrm{b}$ ) pure tungsten cathode with a radius of $1 \mathrm{~mm}$. The profile of the temperature is more broadened and the arc attachment area is larger for the thinner electrode.

Melting of the electrodes and metal vapour are not considered in the model, which leads to temperatures higher than the melting point of tungsten. The normal current density at the cathode tip is $10 \%$ higher in the case of doped tungsten instead of pure tungsten. Figure 5 presents the components of the heat fluxes from the boundary layer to the cathode along the cathode surface for an arc current of $150 \mathrm{~A}$. The

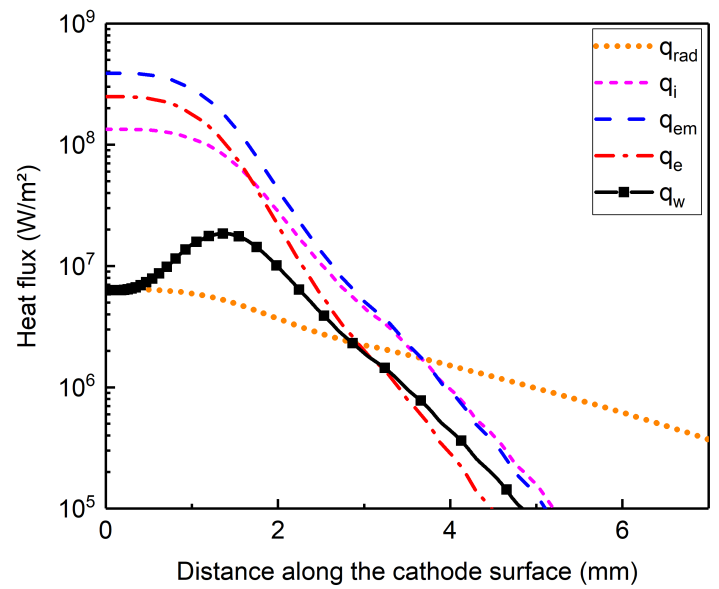

Figure 5. Total heat flux from the plasma to the cathode surface, its components and the contribution of the black body radiation along the cathode for an arc current of $150 \mathrm{~A}$. 

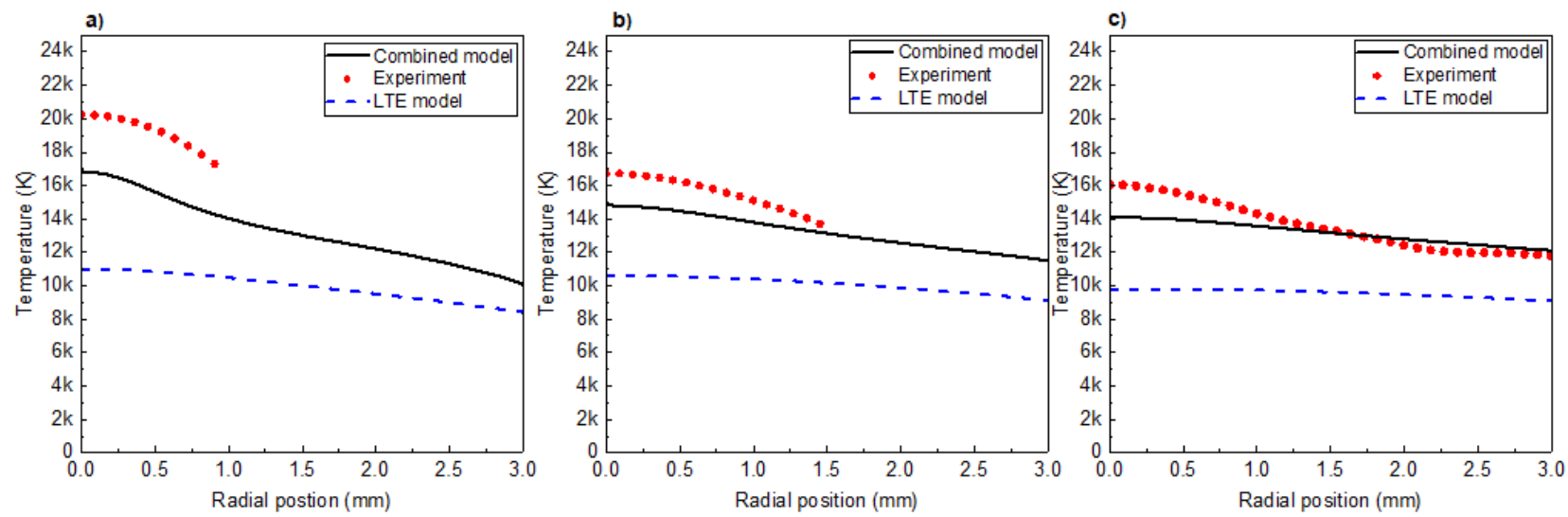

Figure 6. Temperature predicted by the combined model, LTE model and plasma temperature from optical emission spectroscopy at axial position $1 \mathrm{~mm} \mathrm{a),} 2.5 \mathrm{~mm} \mathrm{~b}$ ) and $4 \mathrm{~mm} \mathrm{c}$ ) below the cathode. Arc current is $210 \mathrm{~A}$.

solution shows that the dominant contribution at the cathode tip is that of the emitted electrons $q_{\mathrm{em}}$, which exceeds those of the back diffusion electrons $q_{\mathrm{e}}$ and ions $q_{\text {i }}$ contributions. From the position of $2 \mathrm{~mm}$ on, the main contributions are those of ions and emitted electrons. The resulting flux $q_{\mathrm{w}}$ around the cathode tip is lower than the other components, but still exceeds the contribution of the black body radiation $q_{\text {rad }}$.

The temperature predicted by the combined model in comparison with the LTE model and the values obtained from OES are shown in Figure 6. The results are presented for an arc current of $210 \mathrm{~A}$ and an arc length of $5 \mathrm{~mm}$ at three axial positions: $1 \mathrm{~mm}, 2.5 \mathrm{~mm}$, and $4 \mathrm{~mm}$ away from the cathode tip. One can see that the radial distribution obtained by means of the combined model results in a more focused arc and in higher plasma temperatures near the cathode tip. This modelling approach provides a better agreement with the data obtained by emission spectroscopy.

\section{Conclusions}

The LTE model of the arc plasma is coupled with a model of the cathode boundary layer and is applied to an atmospheric pressure arc in argon. The consideration of the cathode boundary layer allows a more realistic description of the heat and current transfer in the electrodes and the plasma. Therefore, a similar approach has to be applied for $\mathrm{CO}_{2}$. The model results are compared with experimental values and reasonable agreement is obtained.

\section{Acknowledgements}

Financial support of the German Academic Exchange Service (DAAD) is highly acknowledged (Grant No. 91644228).

\section{References}

[1] A. Shirvan et al. Effect of cathode model on arc attachment for short high-intensity arc on a refractory cathode. J. Phys. D: Appl. Phys., 49(48):5201, 2016. doi : 10.1088/0022-3727/49/48/485201/meta.
[2] M. Lisnyak et al. Numerical modelling of high-pressure arc discharges: matching the LTE arc core with the electrodes. J. Phys. D: Appl. Phys., 50(31):5203, 2017. doi:10.1088/1361-6463/aa76d3/meta.

[3] J. J. Lowke et al. A simplified unified theory of arcs and their electrodes. J. Phys. D: Appl. Phys., 30(14):20332042, 1997. doi:10.1088/0022-3727/30/14/011.

[4] L. Sansonnens et al. Prediction of properties of free burning arcs including effects of ambipolar diffusion. $J$. Phys. D: Appl. Phys., 33(2):148-157, 2000. doi: 10.1088/0022-3727/33/2/309.

[5] M. Baeva et al. Two-temperature chemically non-equilibrium modelling of transferred arcs. Plasma Sources Science and Technology, 21(5):5027, 2012. doi:10.1088/0963-0252/21/5/055027.

[6] M. Baeva et al. Novel non-equilibrium modelling of a DC electric arc in argon. J. Phys. D: Appl. Phys., 49(24):5205, 2016. doi : 10.1088/0022-3727/49/24/245205/meta.

[7] A. B. Murphy. Transport coefficients of air, argon-air, nitrogen-air, and oxygen-air plasmas. Plasma Chem. Plasma Proc., 15(2):279, 1995. doi: 10.1007/BF01459700.

[8] A. B. Murphy and C. J. Arundell. Transport-Coefficients of Argon, Nitrogen, Oxygen, Argon-Nitrogen, and Argon-Oxygen Plasmas. Plasma Chem. Plasma Proc., 14(4):451-490, 1994. doi:10.1007/BF01570207.

[9] J. R. Stallcop at al. Potential energies and collision integrals for interactions of carbon and nitrogen atom. J. Thermophys. Heat Transf., 14:480-488, 2000. doi: 10.2514/2.6570.

[10] M. S. Benilov and A. Marotta. A Model of the Cathode Region of Atmospheric-Pressure Arcs. J. Phys. D: Appl. Phys., 28(9):1869-1882, 1995. doi:10.1088/0022-3727/28/9/015/meta. 
[11] M. S. Benilov and M. D. Cunha. Heating of refractory cathodes by high-pressure arc plasmas: I. $J$. Phys. D: Appl. Phys., 35(14):1736-1750, 2002. doi:10.1088/0022-3727/35/14/314.

[12] S. Lichtenberg et al. The plasma boundary layer of HID-cathodes: modelling and numerical results. $J$. Phys. D: Appl. Phys., 38(17):3112-3127, 2005. doi:10.1088/0022-3727/38/17/S13.

[13] J. Heberlein et al. The anode region of electric arcs: a survey. J. Phys. D: Appl. Phys., 43(2):3001, 2010.

doi : 10.1088/0022-3727/43/2/023001/meta. 